\title{
XXXIII. On the objective reality of combination tones
}

\author{
A. W. Rücker M.A. F.R.S. \& E. Edser A.R.C.S.
}

To cite this article: A. W. Rücker M.A. F.R.S. \& E. Edser A.R.C.S. (1895) XXXIII. On the objective reality of combination tones, Philosophical Magazine Series 5, 39:239, 341-357, DOI:

10.1080/14786449508620728

To link to this article: http://dx.doi.org/10.1080/14786449508620728

曲 Published online: 08 May 2009.

Submit your article to this journal $[\pi$

Џ Article views: 3

Q View related articles $\square$

Citing articles: 2 View citing articles 5 
TABLE of the principal Symbols used.

The suffix 1 refers to the dielectric; the suffix 2 to the plate. In our case, where all media are assumed to be non-magnetic,

$$
\begin{aligned}
& \beta=\text { dielectric constant, } \\
& \alpha=4 \pi \times \text { conductivity. }
\end{aligned}
$$

$b=$ ratio of the reflected to the incident amplitude at the first surface of the plate.

$e=$ the corresponding ratio at the second surface.

$c=$ ratio of the refracted to the incident amplitude at the first surface.

$f=$ the corresponding ratio at the second surface.

$d=$ thickness of slab.

$\lambda_{1}=$ wave-length in dielectric.

$\lambda_{2}=$, electrolyte.

$-\psi=$ the change of phase on reflexion at the first surface.

$-\psi^{\prime}=\quad, \quad, \quad$ refraction through the second surface.

$p=-p_{1}+i p_{2}$ is defined by (4).

$\eta, x$ are defined by $(9)$.

$\chi$ gives the rate of damping of the wave-train, being $90^{\circ}$ for a steady ray.

$\rho, \theta$ are defined by (10).

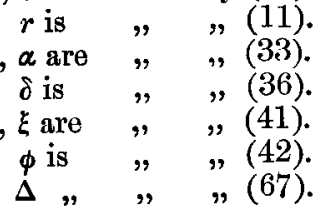

$$
\begin{aligned}
& \mathrm{I}_{t} / \mathrm{I}_{0}=\frac{\text { intensity of transmitted ray }}{\text { intensity of incident ray }} . \\
& \mathrm{I}_{r} / \mathrm{I}_{0}=\frac{\text { intensity of reflected ray }}{\text { intensity of incident ray }}
\end{aligned}
$$

XXXIII. On the Objective Reality of Combination Tones. By A. W. RÜCKER, M.A., F.R.S., and E. EDSER, A.R.C.S.*

T WHE question of the objective existence of combinationtones has been keenly disputed. At first they were regarded as produced within the ear itself. Von Helmholtz in part adopted this view, and gave a theoretical explanation of the way in which the construction of the ear might lend itself to such a result. ('Sensations of Tone,' App. XII.) $\mathrm{He}$ also believed that they existed objectively when the

* Communicated by the Physical Society : read March 22, 1895.

Phil. Mag. S 5. Vol. 39. No. 239. April 1895. 2 A. 
amplitudes of the vibrations of the notes which give rise to them are so great that powers higher than the first have to be considered. He supported this view by mathematical demonstrations, and stated ('Sensations of Tone', transl. by A. Ellis, p. 157) that he had proved their objective existence by making membranes and resonators to respond to combinational tones produced by the siren and harmonium. These views and statements have been adversely criticised by König, Bosanquet, and Preyer. A very lucid account of the controversy was given by the late Mr. Ellis in his translation of the Tonempfindungen, and the net result of the impression produced in his mind is shown by two notes on pages 156 and 157. He there states that the result of Mr. Bosanquet's and Prof. Preyer's experiments is to show that the combinational tones are produced in the ear itself, and that it is probable that the apparent reinforcement of the resonators noticed by Helmholtz arose from imperfect blocking of both ears when using them.

These statements were unqualified, and no condition was made as to the way in which the combination-tones were produced. Helmholtz, for reasons which we need not recount, regarded the siren as the best instrument for producing objective combination-tones ; and we recently determined to submit the question of their existence, which seemed to be decided against him, to another experimental test.

In this paper we give the result of our investigations, as far as they have at present been carried out. We do not regard them as complete, but they at all events prove that when the conditions under which we experimented are fulfilled, there can be no doubt that difference and summation-tones are produced which are capable of disturbing resonating bodies.

The resonator employed in the first instance was a tuningfork. It is well known that this instrument is relatively difficult to excite by resonance, and it was therefore necessary to use an extremely delicate method of detecting whether it was set in motion. For this purpose a mirror attached to one of the prongs was made one of a system by which Michelson's interference-bands were produced. A movement of the prong amounting to half a wave-length of light (say 1/80,000 of an inch) would alter the length of the path of one of the interfering rays by a wave-length. A periodic vibration of this amplitude would cause the band to disappear.

It is therefore evident that an extremely minute movement could be detected. It was at first open to question whether the apparatus would not be so sensitive to accidental dis- 
turbances as to be untrustworthy. This difficulty has, however, been entirely overcome.

A plan of the apparatus is shown in fig. 1. The shank of the tuning-fork, $F$, is imbedded in a mass of lead. One of

Fig. 1.

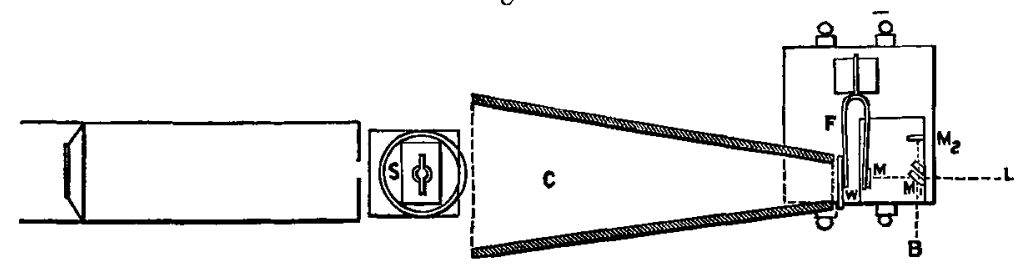

the prongs carries a mirror, M. To the other is attached a square of wood, $W$, of larger area but of the same weight as the mirror. A Lissajous' figure (an ellipse) was formed by reflexion from the mirror and from a small square of silvered glass attached to one of König's large forks adjusted to give the $\mathrm{C}$ of 64 complete vibrations per second, and the pitch of $\mathrm{F}$ was adjusted until only one beat occurred in two minutes. The pitch was thas very accurately known. A double siren, S, was placed between a large König resonator tuned to 64 vibrations and a wooden cone or pyramid, $\mathrm{C}$. The end of the cone was placed about half an inch from $W$, which was rather larger than the narrow end of the cone. The sensitiveness of the apparatus depended in part upon the distance between $W$ and $C$. If the distance was too large, the sensitiveness was diminished. If it was too small, the instrument was unduly affected by chance puffs of air even when not periodic in character.

A source of light, L, was used to form a system of interference-bands by means of the half-silvered mirror $M_{1}$, and the two mirror's $M$ and $M_{2}$. The two interfering rays travelled over the paths $L M_{1} M_{2} M_{1} B$ and $L M_{1} M M_{1} B$ respectively. The distances $M M_{1}$ and $M_{1} M_{2}$ were approximately equal; and since when soda-light was used a change in the length of either of the paths of $\frac{1}{2} \times 589 \mu \mu$ would cause a dark band to shift into the position previously occupied by the next bright band, and since, further, any movement of $\dot{M}$ altered the length of the path of the ray by twice its own magnitude, it is evident that a movement of $\frac{1}{4} \times 589 \mu \mu$, or, say, of one hundred thousandth of an inch, could be easily detected.

The fork $\mathrm{F}$ and the mirrors $\mathrm{M}, \mathrm{M}_{1}$, and $\mathrm{M}_{2}$ rested on a square stone, which was suspended by wires and india-rubber 2 A 2 
door-fasteners from a heavily weighted beam, which itsel rested on india-rubber balls placed at a convenient height on a double pair of wooden "steps." With these precautions it was found that the bands remained tolerably steady even by day when persons were moving about the building, and when the traffic on the frost-bound road produced considerable mechanical vibrations. Even under these conditions, we have satisfied all who have seen the apparatus of the reality of the phenomena. The experiments on which we rely, however, were made on several occasions between midnight and 2 or 3 A.M. The bands were then absolutely clear and steady. They were undisturbed for many minutes at a time when the bellows were being worked and the siren was sounding loudly. It was only when a note of 64 vibrations per second was directly or indirectly produced that they vanished, and there could be no possible doubt or mistake as to whether the disturbance was or was not produced by the sound or combination of sounds under investigation.

Up to the present we have used the fork above described only. It was chosen because it was fairly stiff, and as removable metal mirrors for the production of Lissajous' figures were attached to its prongs, it was possible to replace them by the glass mirror and square of wood without altering its pitch. It would be quite possible to use properly made forks of higher pitch as resonators, and the steadiness of the bands at night is so remarkable, that we believe that if the apparatus were set up in the country, on a stone isolated from the rest of the room, the degree of sensitiveness we have attained could be far surpassed.

\section{Tuning the Siren.}

Three methods were used for determining when the siren was producing the required notes. When one of these was fairly high, the beats given by it and a standard fork were noticed, and the note could thus be kept hovering about the required pitch for a considerable time. Although with the aid of König's large forks we could apply this method to vibration-frequencies of 48 per second and upwards, it was difficult when the notes were very low to recognize the beats with sufficient certainty.

The prongs of various tuning-forks were therefore furnished with two pieces of tin-foil, which opened and closed a slit made in them twice in every complete vibration. They were also compared with a standard by the aid of a revolving cylinder, and were adjusted by weights to the required frequencies. These were so selected as to make one of the 
circles of holes on the siren appear stationary when viewed through the slit, if the velocity of rotation was such that the desired note or notes would be given by the same or other circles of holes. An observer watching the siren through the slit and pressing lightly on the axis with a straw, was able to adjust its speed so that the required combination of sounds was produced for several, and in some cases for many seconds at a time.

We have also projected the image of the row of holes on a screen, the cone of light passing at its narrowest part through the slit in the tinfoil screens carried by the auxiliary fork. When the upper siren was to be used, two semicircles of light mirror-glass were laid on the disk of the lower siren to which the two halves of a paper cog-wheel were fastened. The image of the cogs could thus be projected.

The third method of determining the speed of the siren depended on the use of an instrument first devised by Lord Rayleigh in 1880. A mass of air enclosed in a tube is excited by resonance, and the fact of the excitation is indicated by a light mirror set where the motion is greatest, and inclined at $45^{\circ}$ to the direction of the air-currents. In accordance with the general law that a lamina tends to place itself perpendicular to the direction of a stream, the mirror moves when the air vibrates. In the original apparatus the amount of the movement was controlled by magnets. Since that date Professor Boys has modified the instrument by substituting a quartz-thread suspension for a silk fibre, and using the torsion of the thread instead of the directing force of the magnets. $\mathrm{He}$ exhibited the apparatus during a lecture delivered before the British Association in Leeds. It may be called a mirror-resonator.

Professor Boys has been good enough to make two of these instruments for us, and it was decided that one of them should respond to 161 vibrations per second. The movement of the spot of light reflected from the mirror informed us when the siren was giving this note, and this fact was utilized in one of the experiments.

\section{Sensitiveness of the Apparatus.}

The results were in general improved by laying a small strip of dry blotting-paper upon the prongs. Mere accidental disturbances died out more quickly, and the vibrations of the fork diminished more rapidly, when the notes which had produced them ceased to sound.

A forced movement could be produced in the fork by 
blowing directly down the collecting cone, but a small organ-pipe could be sounded, or a König's fork bowed near the opening without producing the least effect if the frequency of the note produced was not near 64 per second. In like manner many notes of two reed wind-instruments, somewhat similar in construction to the harmonium, were sounded simultaneously so that the room echoed with discordant sound, yet without producing the least effect on the steadiness of the bands.

On the other hand, when one of König's forks tuned to 64 vibrations was touched with the india-rubber covered handle of a gimlet, or was struck with a piece of gas-tubing so lightly that an observer with his ear close to the fork could not detect the fundamental note, the bands instantly disappeared.

It was therefore evident that the apparatus could respond to a vibration which was quite inaudible to a person standing by the resonating fork.

When the König fork was weighted so as to give 63.5 vibrations per second, the bands appeared and disappeared at regular intervals of two seconds, thus corresponding to the beats between the exciting and resonating forks. The bands were not disturbed when the beats exceeded 2 or 3 per second unless the exciting fork was bowed very violently. In the experiments with fainter tones, the resonating fork was only disturbed when the frequency of the exciting note agreed very exactly with its own.

As the speed of the siren was gradually increased from rest the bands always disappeared two or three times, the disturbance being no doubt caused by the upper partials as the vibration-frequency passed, in turn, the values $16,21 \cdot 3$, and 32 per second. No exact measurement was made of the corresponding frequencies, as there could be no question as to whether the partials were produced or as to whether the apparatus could detect them. No such disappearance occurred when the note was higher than $C$, except in the case of certain spurious effects referred to below.

All the experiments were performed several times on each occasion, and on two occasions at least. The bands were sometimes produced by soda-light, and watched by an observer through a telescope.

Sometimes the electric light was used. The bands could then be projected on a screen side by side with the image of the holes or cogs. In this way information as to the note produced and as to the behaviour of the bands was simultaneously conveyed to all who watched the experiments. 


\section{Precautions.}

It will not be thought remarkable that so delicate an apparatus requires careful use.

In the first place, it was necessary to be sure that the disturbance ascribed to the joint action of two notes was not produced by one or both of them when acting alone. In all cases, therefore, in which it was possible to determine the pitch of both notes separately, each note was sounded alone and carried slowly past the particular pitch at which the existence of a combination-tone was to be investigated. In general, no effect was produced; but after the apparatus had been in use for some little time, we were troubled by the fact that two notes which did not differ much from 256 and 320 vibrations respectively disturbed the bands when they were produced separately.

That these effects were spurious was evident from two facts. Firstly, the same notes had previously been sounded for a long time with the special object of determining whether they produced any effect, and had produced none. Secondly, the disturbances were evidently forced. When the fork has been disturbed by a vibration of frequency 64 , it continues to oscillate for several seconds after the disturbing note has ceased. The bands alternately appear and disappear several times before the final state of rest is attained. In the case of a "spurious" effect, the bands reappear instantaneously with absolute clearness the moment the disturbing note ceases, thereby indicating a forced vibration. It was, however, a more difficult task to discover the cause than to determine the character of these disturbances. Finally, it was found that since the apparatus was first set up some cracks had opened in the wooden pyramidal collector. A tap on the side of this will disturb the bands; and when one of the cracked sides was loaded with weights the disturbance due to the 320 note was much reduced. The cracks were then cut out, the defects made good; and since that time this disturbance has entirely ceased.

The effect produced by the 256 note was due to another cause. When the pyramid was removed, the open ends of the resonance-boxes of various tuning-forks $(256,320,384)$ were held near the square of wood which was attached to the fork $F$. It was found that the 256 fork was the only one which affected the bands, and that it only produced any result when the sounding-box was held near that part of the wood which projected beyond the end of the prong. It was therefore 
evident that the two ribs attached to this were not sufficient to prevent a certain flapping of the projecting part, and that this responded to a note of about 256 vibrations. The end of the collecting pyramid was then shifted a little so that the waves of sound impinged only on the more rigid part of the wooden square, and the spurious effect immediately and completely disappeared.

()f the notes which caused these troubles, the lower one was decidedly lower than the $c^{\prime}$ of 256 ; the other corresponded very accurately with the $e^{\prime}$ of 320 . The fact that both these numbers are multiples of 64 may legitimately give rise to the doubt whether the fork $\mathrm{F}$ can be set in motion by disturbances which are multiples of its own frequency.

Even if it be admitted that such forced vibrations would be especially easy to produce, we must insist-(1) that we can distinguish (as above described) between forced and natural vihrations ; $(2)$ that when the apparatus was properly arranged such notes could be produced by the siren for long intervals of time without the least effect on the bands; (3) that two tuning-forks, of 256 and 320 vibrations respectively, have been placed within the wooden pyramid with their sounding-boxes resting on and supported by, the lower side, yet no effect was produced on the bands even when they were both sounding loudly at the same time; (4) that one of König's large forks, with a frequency of 128 , was placed in front of the collecting cone and produced no effect when sounding loudly, though the slightest tap on the 64 fork caused the bands to vanish for many seconds; (5) that the 320 and 384 forks produced no effect when the pyramid was removed and they were held as above described close to the receiving prong of the fork ; $(6)$ and, lastly, that in two out of the five experiments on the difference-tones, and in ali those in which the interference apparatus was used to detect summation tones, the frequencies of the notes employed were neither multiples nor sub-multiples of 64 .

As in all the above experiments the resonator was in position, they also sufficed to prove that tones of 64 vibrations were not manufactured in it by the primary notes when acting singly.

If the objection is raised that, although neither note disturbed the bands when sounded alone, the effect might be due to the double disturbance produced by the two sounds, we think it sufficient to answer that we have always carried the two notes above the pitch at which the difference-tone might be expected to affect the instrument, then lowered "the pitch again till the notes were too flat, and repeated this 
operation several times. The disturbances were only produced when the pitch was nearly, or very nearly, correct. As soon as a few beats per second were heard the bands became visible.

Having thus described the various tests which were applied to the apparatus, we may proceed to describe the experiments without dwelling further on the necessary precautions. It is sufficient to say once for all that they were adopted in each case.

The main object of the enquiry was to obtain evidence as to the objective reality of the combination-tones, and for this purpose the following experiments were arranged.

Each box of the siren had four circles of holes which could be used separately or together. The number of openings in the upper box were $9,12,15$, and 16 , and in the lower 8,10 , 12 , and 18. It will be convenient to refer to these as the 9 row of holes, and so on.

\section{Experiment I.}

The 12 and 15 rows of holes in the upper box were opened, and the pitch was raised until the upper note gave slow beats with a fork of 320 vibrations per second. The lower note was then the $C$ of 256 vilbrations. The difference or beat tone of 64 vibrations affected the instrument powerfully. The experiment was tried both by night and by day. It was difficult to keep the siren exactly at the true pitch, but when the beats were very slow the bands continually disappeared, sometimes for many seconds at a time, then appeared for a moment and then disappeared again. As soon as the pitch was lost by a fow beats per second, the bands remained steady and clearly visible.

The experiment was repeated with the 9 and 12 rows of holes. When the upper note was ( of 256 vibrations, the lower note was 192 vibrations. The difference-tone of 64 vibrations affected the fork very powerfully.

The experiment was also modified by opening the 10 and 12 rows of holes. When the notes corresponded to 320 and 384 vibrations respectively, the bands disappeared as before.

\section{Experiment II.}

In experiment $I$. the frequencies of the difference-tone and of König's first lower beat-tone were identical. The experiment was therefore varied by using 8 and 18 rows of holes. The frequency of the difference-tone was thus proportional to 10 , while that of König's lower beat-tone would be $18-2 \times 8=2$. When the siren was revolving at the rate of 
6.4 revolutions per second, the two notes corresponded to 51.2 and 115.2 vibrations per second, the difference-tone being 64 .

The pitch was determined on different occasions by different methods. Firstly, by noting the beats between the higher note and a König's fork adjusted to $115 \cdot 2$ complete vibrations per second, and secondly, by watching the row of eight holes through a slit carried by a fork which gave $\mathbf{2 5 . 5}$ vibrations per second.

The effect was rather feebler than in the last experiment, but there was absolutely no doubt as to the objective reality of the difference-tone. The bands regularly disappeared when the required pitch was obtained, and reappeared when it was lost.

Again the 15 and 9 rows of holes were used. The difference-tone is thus proportional to 6, and König's beat-tone to $9 \times 2-15=3$. When the rate of revolution was $10 \cdot \dot{6}$ the two notes were 160 and 96 respectively. In this experiment the mirror-resonator which responds to 161 vibrations was employed to determine the rate of the siren. The bands and the spot of light were sometimes watched together: on another occasion one observer who could not see the bands raised his hand whenever the spot of light moved. The bands invariably disappeared at the instant that this signal was made.

\section{Experiment III.}

The next experiment was directed to determine the objective reality of König's lower beat-tone when the interval was greater than an octave. The 8 and 18 rows of holes being kept open as before, the speed was increased until the lower note was that of 256 vibrations. The upper note was then 576 , and König's lower beat-tone was of $576-2 \times 256=64$ vibrations.

We lay less stress on negative than on positive results ; but we tried for a long time on two occasions to get evidence of the objective character of the note, but entirely failed. The pitch was determined by the beats with a 256 fork.

\section{Experiment IV.}

We next turn to observations on the summation-tone. The 8 and 10 rows of holes were opened, so that when the cover made 3.55 revolutions per second the summation-tone would be that of $18 \times 3 \cdot 5=64$ vibrations, 
The pitch of the notes given by the siren was again determined in different ways on different occasions. The summation-tone being produced in the lower box, the 15 row in the upper box was also opened, thus producing a note of $15 \times 3 \cdot \dot{5}=53 \cdot \dot{3}$ vibrations per second. The required speed was determined by making the beats vanish between this note and a König's fork tuned to give 53.3 vibrations. With this method it was difficult to keep the speed constant for a length of time sufficient to disturb the resonating fork appreciably. When the pitch was altered very slowly the bands disappeared just as the right note was reached, and did not disappear at any other time during the experiment.

On another occasion the 9 and 12 rows of holes were opened, so that the summation-tone of 64 vibrations would be given when the siren made $3 \cdot 05$ revolutions per second. The is row of holes was watched through a fork of $27 \cdot 2$ vibrations, so that 54.4 views would be obtained while a hole moved over $18 \times 3.05=54.9$ intervals. Hence the right pitch was obtained when the holes moved slowly forwards. The bands invariably disappeared when this state of things was attained.

On a third occasion the lower cover of the siren was covered with a thin piece of silvered glass as above described, carrying a concentric circle of black paper, the edge of which was divided into 18 equidistant cogs. An image of these was produced on a screen by a lens, and made intermittent by the 27-vibrations fork. The disturbance due to the summation-tone was again and again made evident when the images of the cogs appeared to be moving slowly. In the intervals the bands were beautifully steady.

The earlier of these experiments were performed before, and the later ones after, the apparatus had been taken down and set up again in another room. They left in the minds of those who saw them no shadow of doubt as to the objective reality of a note corresponding in frequency with the summationtone.

We now turn to experiments intended to throw light on the cause of the production of this note.

\section{Experiment V.}

It has been suggested that the summation-tone may be the difference-tone of partials. König (Acoustique, p. 127) remarks that it may occasion some surprise that the particular harmonics whose difference-tone corresponds to the summation-tone should be especially prominent; but he points out 
that in some cases the difference-tones of the lower harmonics correspond either to the fundamentals or to some of their upper partials. In the case of the fourth $(3: 4)$, however, Konnig remarks that the 5 th partials would give a difference-tore (5) which could be distinguished from the lower partials, and that the difference-tone of the 7th partials would give the summation-tone. Now we have already proved (Exp. IV.) that the summation-tone produced by two notes separated by the interval of a fourth $(9: 12)$ is objective; and if this is due to the difference-tone of the 7th partials, there seems to be no reason why the difference-tone of the 5th partials should not be objective also, and probably more intense.

We therefore increased the velocity of revolution to $4 \cdot 27$ per second, the 9 and 12 rows of holes being opened as before. The frequencies of the two notes were thus $38 \cdot 43$ and $51 \cdot 24$. The pitch was determined by keeping the 12 holes nearly stationary when viewed 51 times a second by aid of the 25.5 fork. The first difference-tone was $12 \cdot 81$, and the differencetone of the 5th partials was 64:05. When the speed corresponding to this difference-tone was attained there were occasional flickers of the bands, so that it is possible that it has an objective existence. But, on the other hand, the effect was less than that produced by the summation-tone. The bands never disappeared for any considerable length of time, as they did when the fork responded to the summation-tone, and the experiment left no donbt in our minds that the greater effect was produced by the summation-tone.

\section{Experiment VI.}

The same point was also investigated in another way. If the summation-tone of two notes of frequencies $p$ and $q$ corresponds to the difference-tone of the $n$th partial, we must have

$$
(p+q)=n(p-q)
$$

where $n$ is an integer. If, however, the 9 and 16 rows of holes were opened,

$$
p+q=25, \quad p-q=7
$$

so that the summation-tone could not be produced by partials of the same order. The 10th partial of the higher note beating with the 15 th of the lower note $(160-135=25)$ would indeed have the same frequency as the summation-tone, but it appears to us absurd to suppose that so improbable a combination should produce appreciable results. It is true that lower partials may give beat-tones near to the summation-tone. 
Thus $5 \times 16-6 \times 9=26$. But if we are to assume that any pair of partials can thus produce objective tones, the number of combinations will be so great that the fork ought to have been disturbed frequently when the note of the siren was being raised to the required pitch. As a matter of fact, when once the $\mathbf{C}$ of 64 vibrations was passed, so that all the partials were higher than the pitch of the resonating fork, no such disturbances were ever observed except when the difference- or summation-tone of the primaries was produced. Putting, therefore, all such fantastic combinations aside, the experiment may be regarded as a test whether the summation-tone can be produced when it cannot be due to two partials of the same order.

When the velocity of revolution was $2-56$ per second, the 16 and 9 holes gave notes of 40.96 and 23.04 vibrations. The sum of these is 64 . The 12 holes were viewed through a slit alternately closed and opened by a fork of 15 vibrations per second, and when the holes appeared to move slowly the summation-tone caused the bands to disappear.

In this experiment, however, the third partial of the lower note corresponds to $69 \cdot 12$ vibrations, and we thought it desirable to make sure that the disturbance attributed to the summation-tone was not in reality due to this partial. This was the more important, because the difference in the speeds of the siren when the summation-tone and the partial in question corresponded to 64 vibrations was very small.

Thus, when the speed was 2.56 revolutions per second each of the 12 holes would advance through 30.72 intervals in a second, and since the fork gave 30 views per second the holes would appear to move slowly forwards.

When the speed was $2 \cdot 37$ revolutions per second the third partial of the lower note (9 row of holes) would be $3 \times 9 \times 2 \cdot 37=64$, and each hole of the 12 rows would advance through 28.44 intervals - that is, would appear to recede through 1.56 intervals per second. Thus the partial would be most efficient in promoting disturbance when the holes appeared to go backward with moderate speed.

The question to be answered was whether these two disturbances could be confused with each other.

When care was taken to keep the pressure in the windchest the same whether one or both sets of holes were opened, the effect of the partial produced by the 9 set of holes could hardly be detected. The bands were shaken a little when the row of 12 holes appeared to move backwards, but they did not disappear ; whereas they were completely wiped out by the summation-tone when the two notes were sounded. 
When the pressure on the wind-chest was increased, the rate of revolution being nevertheless maintained constant by pressing lightly on the axle of the siren with a straw, the effect of the partial was more marked, but it was always produced when the holes appeared to move backwards.

On the other hand, when both notes were sounded together and when the pitch was gradually reduced to the desired point, the disturbance always began when the holes moved slowly forwards. If the pitch fell very slowly it was possible to note a reduction of the disturbance, followed by an increase when the holes appeared to move backwards.

We thus convinced ourselves that the effects of the two sources of disturbance could be distinguished, and that the supposed summation-tone was not due to the partial of the lower note.

\section{Experiment VII.}

We have also succeeded in demonstrating the reality of the summation-tone with a mirror-resonator constructed by Professor Boys to respond to a vibration-frequency of 576.

The rows of 15 and 12 holes being opened, notes of 320 and 256 vibrations were produced. When they were sounded separately, the mirror moved slightly. When they were sounded together, the spot of light was driven off the scale when the upper note coincided with that of a 320-vibration fork, but immediately returned when this pitch was lost.

The experiment was varied by using the 16 and 12 rows, and also the 16 and 9 rows. The summation-tone corresponds to 576 vibrations when the upper note is of $329 \cdot 15$ and 360 vibrations in these two cases respectively. The 320-fork was used, and the disturbance occurred in the one case when the pitch of the note was nearly the same as before, and in the other when it was about a tone higher.

We attach great importance to this corroboration of our results by an instrument of a totally different construction from that first employed.

The attempt to obtain proof of the existence of a differencetone by means of the mirror-resonator of 161 vibrations has not been successful. The instrument is much less affected by the note to which it responds than is that which answers to 576 vibrations, even when that note is produced directly by the siren. It is, therefore, perhaps not wonderful that it gives no reliable evidence of the existence of a differencetone.

We now sum up the results we have obtained in two tables. 
Objective Reality of Combination Tones.

Instrument. Interference Resonator.

\begin{tabular}{|c|c|c|c|c|}
\hline $\begin{array}{l}\text { Number of } \\
\text { holes in siren }\end{array}$ & Interval. & Frequeneies. & $\begin{array}{c}\text { Combination- } \\
\text { tone. }\end{array}$ & $\begin{array}{c}\text { König's } \\
\text { Beat-tone. }\end{array}$ \\
\hline 12 and 10 & Minor Third. & $384 \quad 320$ & 64) & 64 \\
\hline 15,12 & Major Third. & $320 \quad 256$ & 64 & 64 \\
\hline 16,12 & Fourth. & $256 \quad 192$ & 64 & 64 \\
\hline 15 & Major sixth. & 160 & 64 & 32 \\
\hline 18,8 & $\left\{\begin{array}{l}\text { Octave and } \\
\text { major tone. }\end{array}\right\}$ & $115 \cdot 2 \quad 51 \cdot 2$ & 64 & $12 \cdot 8$ \\
\hline 10 and 8 & Major Third. & $35 \cdot \dot{5} \quad 28 \cdot \dot{4}$ & 64 & \\
\hline $12 "$ & Fourth. & $\begin{array}{lll}36 \cdot 57 & 27 \cdot 43\end{array}$ & 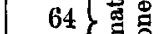 & \\
\hline 16,9 & Minor Seventh & $40.96 \quad 23.04$ & 64 蒠 & \\
\hline
\end{tabular}

Instrument. Mirror Resonator.

Summation-Tones.

\begin{tabular}{|c|l|ll|c|}
\hline $\begin{array}{c}\text { Number of } \\
\text { holes in siren. }\end{array}$ & Interval. & Frequencies. & Sum. \\
\hline 15 and 12 & Major third. & 320 & 256 & 576 \\
16,12 & Fourth. & $329 \cdot 15$ & 246.85 & 576 \\
16,9 & Major sixth. & 360 & 216 & 576 \\
\hline
\end{tabular}

Negative Results.

We have tried several times to obtain indications of combination-tones when the primary notes were produced by tuning-forks. Two of König's large forks adjusted to 48 and 112 vibrations produced no effect when bowed simultaneously before the collector, and, as has been stated, smaller forks giving 256 and 320 vibrations have been placed inside the collector when sounding loudly. No effect whatever was produced, and there can be no doubt that if objective combination-tones are produced in such cases they are very much less intense than those generated by the siren.

Experiments have been made with reeds and with organpipes, but up to the present with uncertain results in the first case and negative results in the second. We hope to investigate these cases further. 
We have made several attempts to detect combinationtones of higher orders, such as $2 p+q$ and $2 p-q$, but without success.

\section{Conclusion.}

We may in conclusion refer to some of the suggestions which have been made to account for the combination-tones by theories other than those of Helinholtz.

König's suggestion that they are the beat-tones of upper partials has been discussed and shown to be inadequate to explain the facts of observation.

Again, it has been argued that the summation-tone is the beat-note between the second partial of the higher note (the octave) and the beat-tone of the two primaries. It follows as a malter of algebra that such an explanation must always be numerically correct, for $2 a-(a-b)=a+b$, and our experiments throw no new light on the matter. It appears to us, however, that since propinquity between the sources of sound, causing a violent disturbance, is favourable to the production of combination-tones, while it is not necessary for the production of beats, the facts of experiment are in this case also in favour of von Helmholtz's views.

A still more subtle objection has been taken by Terquem (Annales d'Ecole Normale, 1870, p. 356). When two rows of holes are open in the siren, there may be occasions on which all the holes of both rows are opened simultaneously and others on which only one row is in action at one time. Terquem attempts to calculate the effects of irregularities such as these, but in the first place he specifically refrains from attacking the theory of Helmholtz; - secondly, he does not apply calculation to the siren of Helmholtz; thirdly, he points out that the relatively large size of the holes in that instrument would reduce the effects he predicts ; and, lastly, he admits that his results require confirmation by experiment. Putting these points aside, however, his theory leads to the conclusion that the two notes which we have been regarding as fundamental are reinforced harmonics in a series of which the fundamental note corresponds to the greatest common measure of these frequencies. Both the summation and the difference tone must be included in such a series; but Terquem's theory gives no reason why they should have such exceptional importance as experiment proves that they have. Lastly, as he expressly repudiates the idea that partials have an objective existence (loc. cit. p. 274), and includes the combination-tones in a series of partials, the experiments described by us must on this point be regarded as opposed to his views. 
We think, then, that our experiments prove that von Helmholtz was correct in stating that the siren produces two objective notes the frequencies of which are respectively equal to the sum and difference of the frequencies of the fundamentals, and that our observations are also more or less opposed to the theories by which König, Appun, and Terquem have sought to account for the production of these notes.

We believe that the method we have devised is capable of greater sensitiveness. It can be extended by employing forks of different pitches, and it is quite possible that less massive forks may enable us to detect effects which have hitherto escaped us. We therefore refrain from any wide generalizations until a wider foundation of experiment has been laid.

P.S.- Since the above was written Prof. S. P. Thompson has drawn our attention to a paper by 0 . Lummer, published in $\mathbf{1 8 8 6}$ (Verh. phys. Gesell. Berlin, 1886, No. 9, p. 66), which had escaped our notice, as it is not abstracted in the Beiblätter. Herr Lummer obtained evidence of the objective character of the summation-tone by means of the microphone.

XXXIV. Energy Movements in the Medium separating Electrified or Gravitating Particles. By H. N. Allen, University of Nebraska, Lincoln, $\mathrm{Neb}$.

1. GARADAY and Maxwell have shown that it is possible to look on the potential energy of electric separation as residing in the surrounding dielectric, and that each of the cells, bounded by the walls of a tube of force and two neighbouring equipotential surfaces, can be looked upon as containing a certain definite amount of energy.

This energy-distribution is not in general permanent, and can only be regarded as a step towards some simpler arrangement. Thus a positive and a negative electrified body suspended in space, and acted on only by the electrical forces between them, are never in equilibrium until they are in actual contact. The energy-distribution in the dielectric changes constantly as they approach. Poynting has shown $\uparrow$ how energy is transferred from one point to another in an electromagnetic field; and we are quite accustomed to think of energy as flowing from dynamo to motor through the æther, or from primary to secondary in an alternating-current transformer.

In the following pages an attempt is made to deduce a few

* Communicated by the Physical Society : read March 8, 1895.

† Phil. Trans. 1884, Pt. II. p. 343.

Phil. Mag. S. 5. Vol, 39. No. 239. Aprí 1895. 2 B 